\title{
Ensuring consistent reporting of clinical pharmacy services to enhance reproducibility in practice: an improved version of DEPICT
}

\author{
Inajara Rotta, MSc, ${ }^{1}$ Teresa M. Salgado $\mathrm{PhD},{ }^{3}$ Daniel C. Felix PharmD, ${ }^{4}$ \\ Thais T. Souza MSc, ${ }^{2}$ Cassyano J. Correr $\mathrm{PhD}^{5}$ and Fernando Fernandez-Llimos, \\ $\mathrm{PhD} \mathrm{MBA}^{6}$ \\ ${ }^{1} \mathrm{PhD}$ Candidate, ${ }^{2} \mathrm{PhD}$ Candidate, Post-Graduate Program of Pharmaceutical Sciences, Federal University of Parana, Curitiba, Brazil \\ ${ }^{3}$ Post-Doctoral Research Fellow, Department of Clinical, Social, and Administrative Sciences, College of Pharmacy, University of Michigan, Ann \\ Arbor, MI, USA \\ ${ }^{4}$ PGY2 Transplant Pharmacy Resident, Department of Pharmacy, University of Wisconsin Hospital and Clinics, Madison, WI, USA \\ ${ }^{5}$ Assistant Professor, Department of Pharmacy, Federal University of Parana, Curitiba, Brazil \\ ${ }^{6}$ Assistant Professor, Research Institute for Medicines (iMed.ULisboa), Department of Social Pharmacy, Faculty of Pharmacy, University of \\ Lisbon, Lisbon, Portugal
}

\section{Keywords}

evidence-based practice, outcome and process assessment (health care), pharmaceutical services, pharmacists, reproducibility of results, validation studies

\author{
Correspondence \\ Fernando Fernandez-Llimos \\ Research Institute for Medicines \\ (iMed.ULisboa) \\ Department of Social-Pharmacy, Faculty of \\ Pharmacy \\ University of Lisbon \\ Av. Prof. Gama Pinto \\ 1649-003 Lisboa \\ Portugal \\ E-mail: f-llimos@ff.ul.pt
}

Accepted for publication: 19 January 2015

doi:10.1111/jep.12339

\begin{abstract}
Rationale, aims and objectives DEPICT (Descriptive Elements of Pharmacist Intervention Characterization Tool) was created in response to the frequently reported issue of poor intervention description across studies assessing the impact of clinical pharmacy activities. The aim of this study was to create an improved version of DEPICT (i.e. DEPICT 2) to better characterize clinical pharmacy services in order to ensure consistent reporting, therefore enhancing reproducibility of interventions in practice.

Method A qualitative approach through a thematic content analysis was performed to identify components of pharmacist interventions described in 269 randomized controlled trials. A preliminary version of DEPICT 2 was applied independently by two authors to a random sample of 85 of the 269 RCTs and reliability determined by the prevalenceadjusted bias-adjusted kappa (PABAK) or the intraclass correlation coefficient (ICC). The final version of DEPICT 2 was compared against DEPICT 1.

Results The final version of DEPICT 2 comprised 146 items and 11 domains. The interrater agreement analysis showed that DEPICT presented good to optimal reproducibility, with a mean PABAK value of 0.87 (95\% CI 0.85-0.89) and a mean ICC value of $0.88(95 \%$ CI 0.62-1.14). The mean difference between items checked in the two versions (DEPICT 2 - DEPICT 1) was 10.58 (95\% CI 9.55-11.61), meaning that approximately 11 more components were identified in the new version of DEPICT.

Conclusions DEPICT 2 is a reliable tool to characterize components of clinical pharmacy services, which should be used to ensure consistent reporting of interventions to allow their reproducibility in practice.
\end{abstract}

\section{Introduction}

The literature has repeatedly attempted to demonstrate the impact of clinical pharmacy services in patient health outcomes in several medical conditions through systematic reviews and meta-analyses $[1,2]$. It is common to report study heterogeneity in meta-analyses as a means of assessing the variability among studies, either in terms of participants, interventions, outcomes studied and study design, or with regards to variability in the intervention effects being evaluated in the different studies [3]. In the pharmacy prac- tice literature, several meta-analyses showed heterogeneities over $50 \%$, which is the cut-off above which heterogeneity is considered high [4-6], and in several cases, meta-analysis could not be performed because of the high heterogeneity found among primary studies [7].

In addition to the heterogeneity issue, clinical pharmacy services are also complex health interventions that include a number of interacting components that may act both independently or interdependently to achieve a desired outcome [8]. Thus, isolating these components and determining which of them are the most 
meaningful for the intervention outcome obtained can be troublesome. In geriatrics, previous authors have developed a graphical method to facilitate the reporting of process evaluation's results of complex multi-component interventions focusing on health care interventions for elderly people [9]. The authors argued that such an instrument might aid the critical appraisal of primary studies as well as performing mixed-method systematic reviews of heterogeneous and complex interventions [9].

Another limiting aspect of conducting systematic reviews and meta-analyses in the pharmacy field is that generally the interventions performed by the pharmacist are poorly and inconsistently described in primary studies, as pointed out by numerous authors $[2,10,11]$. In order to address the issue of the lack of an in-depth intervention description, a tool to characterize the components of pharmacist interventions performed as part of clinical pharmacy services - DEPICT (Descriptive Elements of Pharmacist Intervention Characterization Tool) - was recently developed [12]. The tool contained 54 items where each item was designed to reflect components of pharmacists' interventions. DEPICT was created with the aim of allowing the retrospective analysis of published studies, facilitating comparisons among them, but also as a way of assisting authors when reporting pharmacist interventions to ensure their reproducibility in practice [12].

After the experience acquired with the application of DEPICT to studies describing pharmacists' interventions in the management of patients with chronic kidney disease, a need to create an improved version of the instrument arose [13]. In that study, the authors identified several gaps with some interventions that were specific of this setting not being appropriately reflected on the instrument, namely studies describing therapeutic protocol implementation by pharmacists [13]. In addition, DEPICT 1 was developed based on the intervention description available in 49 systematic reviews and not in their respective 269 primary studies, which further contributed to a less detailed description of clinical pharmacy services. Therefore, the aim of the present study was to create an improved version of DEPICT (i.e. DEPICT 2) to better characterize clinical pharmacy services in order to ensure consistent reporting, therefore enhancing reproducibility of interventions in clinical practice.

\section{Methods}

The development of DEPICT 2 was carried out in three phases: (1) creation of a preliminary version of DEPICT 2; (2) achievement of the final version of DEPICT 2; and (3) comparison of the final version of DEPICT 2 against DEPICT 1.

\section{Preliminary version of DEPICT 2}

A qualitative analysis of the description of pharmacist interventions contained in the 269 randomized controlled trials (RCTs) included in the 49 systematic reviews that served as the basis for the creation of DEPICT 1 was performed in September 2012. A thematic content analysis was performed and components of pharmacist interventions described in the 269 studies were coded and ordered by thematic similarity to reduce data to highly conceptualized themes. During the coding process carried out by one of the authors (C.J.C.), a permanent debate was maintained with three other authors (F.F-L., T.M.S. and T.T.S.) to ensure an appropriate grouping of pharmacist interventions. This process resulted in the addition of more items to the original instrument and subdivision of others to better describe the components of the interventions performed as part of clinical pharmacy services. Data analysis was performed with the support of N-vivo 8 software (http:// www.qsrinternational.com).

\section{Final version of DEPICT 2}

The preliminary version of DEPICT 2 was applied independently by two authors (I.R. and D.C.F.) to a random sample of the previous 269 RCTs analysed between October and December 2012. Sample size was calculated using a two-tailed test, an estimate proportion of positive rating for each item of 0.30 , a minimum acceptable value of kappa of 0.70 , a statistical power of $80 \%$, and assuming a null hypothesis value of kappa to be 0.40 . The number of RCTs required to detect a statistically significant kappa value $(P<0.05)$ was thus estimated to be 85 . These 85 articles were selected out of the $269 \mathrm{RCTs}$ using a random number list generator (randomizer.org) and the final 85 articles randomized corresponded to 82 different studies. In order to ensure homogeneity in the analysis of the 82 studies by the two authors, a manual with clear instructions for item interpretation (available at depictproject.org) was developed prior to the assessment.

To assess the reliability of the instrument, the inter-rater agreement kappa coefficient was calculated for all dichotomous variables and the intraclass correlation coefficient (ICC) for discrete or continuous variables. To avoid potential effects of a low component prevalence on kappa results, the prevalence-adjusted biasadjusted kappa (PABAK) was used over kappa [14]. PABAK was calculated using the software WinPepi version 11.25 (http:// www.brixtonhealth.com). As standards for strength of agreement for PABAK coefficient, we assumed $<0=$ poor; $0-0.20=$ slight; $0.21-0.40=$ fair; $0.41-0.60=$ moderate; $0.61-0.80=$ substantial; and $0.81-1.00=$ almost perfect agreement [15]. As standards for strength of agreement for ICC, we adopted cut-offs similar to those used for the kappa statistic, since they represent directly analogous measures: $0-0.2$ = poor agreement; $0.3-0.4=$ fair agreement; $0.5-$ $0.6=$ moderate agreement; $0.7-0.8=$ strong agreement; and $>0.8=$ almost perfect agreement [15] .

After performing the reliability analysis, items with a PABAK or an ICC value under or equal to 0.60 had their interpretation reassessed and wording modified accordingly, and the instructions for item scoring in the manual were adapted. The two authors that performed the reliability analysis were kept blind to this assessment and conducted a subsequent analysis of the RCTs using the modified version of the manual. PABAK and ICC coefficients were then recalculated and, after this second analysis, only items with a PABAK or ICC value above 0.60 were included in the final version of DEPICT 2.

\section{Comparison of the final version of DEPICT 2 against DEPICT 1}

A comparison of the final version of DEPICT 2 was performed against the published version of DEPICT 1 . The same authors that performed the reliability analysis for DEPICT 2 (I.R. and D.C.F.) applied DEPICT 1 independently to the same 82 studies. The number of items checked for a given study when applying both 
DEPICT 1 and DEPICT 2 was compared and the frequency of items contained exclusively in DEPICT 2 was evaluated. The Wilcoxon signed-rank test was used to compare the two versions of DEPICT on the same sample of studies.

\section{Results}

\section{Preliminary version of DEPICT 2}

Following the qualitative analysis of the intervention description in the 269 RCTs, 92 more items were added to the previous 54 in DEPICT 1. The original structure of DEPICT was also modified and the number of domains was reduced from 12 to 11 . While DEPICT 1 was created as a list of items containing a single column to fill out with a yes/no answer, DEPICT 2 contains two columns to encompass interventions targeting both patients/caregivers and health care professionals. In addition, some items in DEPICT 2 can be checked for both patients/caregivers and health care professionals (domains 1,2 and 6 to 10), others can only be checked for patients/caregivers (domain 11), and other items describe elements of the intervention that are independent of the recipient (domains 3, 4 and 5). A set of additional items were included in DEPICT 2 to ensure that none of the domains would remain blank (items 2.10, 3.04, 4.15, 5.16, 6.08, 7.10, 8.09 and 11.01). A summary of the main differences between each version of DEPICT is outlined in Table 1.

\section{Final version of DEPICT 2}

DEPICT 2 preliminary version was ultimately applied to 82 studies given that in three instances, two different articles were part of the same study [16-20]. The studies analysed were published between 1977 and 2009 and were performed in the following countries: United States $(n=50)$, United Kingdom $(n=8)$, Australia $(n=6)$, Canada $(n=4)$, the Netherlands $(n=3)$, Chile $(n=2)$, India $(n=2)$, Belgium $(n=1)$, Brazil $(n=1)$, China $(n=1)$, Germany $(n=1)$, Sweden $(n=1)$, Thailand $(n=1)$ and United Arab Emirates $(n=1)$.

After applying DEPICT 2 by the two raters to the 82 studies, the mean PABAK value obtained for the dichotomous variables was 0.85 denoting an 'almost perfect' agreement (95\% CI 0.82-0.87). The PABAK value was less than or equal to 0.60 for nine items $(6.3 \%)$ and the minimum value obtained was 0.14 . For the remaining 133 items, $40(30.0 \%)$ presented a PABAK value comprised between 0.61 and 0.80 (substantial agreement) and 93 items (70.0\%) between 0.81 and 1.0 (almost perfect agreement). After rewriting the nine items that presented a PABAK equal to or lower than 0.60 and modifying their description in the manual, the recalculated mean PABAK value obtained was 0.87 (95\% CI 0.85 0.89). Finally, 45 items (31.7\%) presented a PABAK value between 0.61 and 0.80 (substantial agreement) and $97(68.3 \%$ ) between 0.81 and 1.0 (almost perfect agreement).

For the discrete/continuous variables, the mean ICC value in the first assessment was 0.46 (95\% CI 0.21-1.14), which can be classified as fair agreement. The ICC value was less than or equal to 0.60 for two items $(50.0 \%)$ and the minimum value obtained was 0.09 . The remaining two items $(50.0 \%)$ presented ICC values of 0.64 and 0.98 (moderate and almost perfect agreement, respectively). After the second assessment, the mean ICC was $0.88(95 \%$ CI 0.62-1.14) and all items presented an ICC value between 0.64 and 0.98 . The overall results of PABAK and ICC for each round of assessment are presented in Supporting Information Appendix S1.

\begin{tabular}{|c|c|c|}
\hline & DEPICT 1 & DEPICT 2 \\
\hline $\begin{array}{l}\text { Source of information } \\
\text { extraction }\end{array}$ & 49 systematic reviews & 269 randomized controlled trials \\
\hline \multirow[t]{2}{*}{ No. items } & 54 & 146 \\
\hline & $\begin{array}{l}\text { (53 dichotomous variables } \\
\text { and } 1 \text { discrete variable) }\end{array}$ & $\begin{array}{l}\text { (142 dichotomous variables and } 4 \\
\text { discrete variables) }\end{array}$ \\
\hline No. domains & 12 & 11 \\
\hline \multirow[t]{14}{*}{ Domain designation } & A. Contact with the patient & 1. Contact with recipient \\
\hline & B. Timing of intervention & 2. Setting \\
\hline & C. Setting of intervention & 3. Focus of intervention \\
\hline & D. Target population & 4. Clinical data sources \\
\hline & E. Clinical data sources & 5. Variables assessed \\
\hline & F. Assessment & 6. Action(s) taken by the pharmacist \\
\hline & G. Pharmacist's autonomy & 7. Timing of action(s) \\
\hline & H. Pharmacist & 8. Materials that support actions \\
\hline & communication & 9. Repetition \\
\hline & I. Support resources & 10. Communication with recipient \\
\hline & provided by pharmacist & 11. Changes in therapy and lab tests \\
\hline & $\begin{array}{l}\text { J. Education and } \\
\text { counselling }\end{array}$ & \\
\hline & L. Follow-up & \\
\hline & M. Other actions & \\
\hline Target of the intervention & Patients & $\begin{array}{l}\text { Patients/caregivers and health care } \\
\text { professionals }\end{array}$ \\
\hline
\end{tabular}

Table 1 Summary of the main differences between DEPICT 1 and DEPICT 2 
At the end of this process, DEPICT 2 conserved the same number of items and domains as the preliminary version, only with minor changes to the wording and to the scoring manual (Appendix A).

\section{Comparison of the final version of DEPICT 2 against DEPICT 1}

Globally, the score obtained by applying DEPICT 2 to the 82 RCTs was higher compared with the score obtained when using DEPICT 1 (24.0 versus 13.4, Wilcoxon signed-rank test, $P<0.001)$. The mean difference between items checked in the two versions of DEPICT (DEPICT 2 - DEPICT 1) was 10.6 (95\% CI 9.6-11.6), meaning that approximately 11 (85\%) more components of pharmacist interventions were identified in the new version of DEPICT. In the 50 interventions targeting patients only, the scores obtained were 18.0 (95\% CI 16.8-19.3) and $11.2(95 \%$ CI 10.0-12.4) when using DEPICT 2 and DEPICT 1 , respectively (Wilcoxon signed-rank test, $P<0.001$ ). For the 11 interventions, where pharmacists established contact with the health care professional alone, the score obtained when using DEPICT 2 was 18.6 (95\% CI 16.3-21.0) and 6.1 (95\% CI 4.0-8.1) when using DEPICT 1 (Wilcoxon signed-rank test, $P<0.001$ ).

\section{Discussion}

Our study aimed to improve the initial version of DEPICT to better describe the components of clinical pharmacy services in order to enhance the reproducibility of interventions in clinical practice by ensuring their consistent reporting. To accomplish this goal, we analysed pharmacists' interventions described in 269 RCTs that were included in the 49 systematic reviews used to develop DEPICT 1, rather than just analysing the description of the interventions from systematic reviews. This allowed the collection of more detailed and complete information on the clinical pharmacy service provided.

A final version of DEPICT 2 was obtained with the revised tool comprising 11 domains and 146 items and including an extensive list of components contemplating all variables that could be part of a complex clinical pharmacy service. Some items were common between the two versions of the tool, but most of the items included in DEPICT 2 arose from a new analysis. The structure of DEPICT 2 was designed to allow the analysis of studies describing pharmacists' interventions targeting both patients/caregivers and health care professionals. After the second reliability assessment, the mean PABAK value obtained reflected an almost perfect agreement and ICC values presented a moderate to almost perfect agreement. These results show that the instrument is reliable and that the manual created to ensure consistency in the analysis served its purpose.

Following the incorporation of new items and the subdivision of pre-existing ones, studies assessed with DEPICT 2 had approximately 11 more components checked to describe pharmacists' interventions, which suggests a higher discriminating ability of this version. This applies to interventions targeting both patients and health care professionals. While there was an evident difference between scores of studies describing interventions targeting only patients or targeting only health care professionals with DEPICT 1 (11.2 versus 6.1), this difference was neutralized with
DEPICT 2 (18.0 versus 18.6). This reinforces the existing need to restructure DEPICT 1 to address its limitation of not including pharmacy services targeting health care professionals alone. Therefore, the creation of DEPICT 2 allowed an equivalent identification of clinical pharmacy service components targeting both patients and health care professionals.

Besides its utility in helping isolate components of clinical pharmacy services involving complex interventions, DEPICT can also be a useful means of identifying specific actions that do not characterize actual clinical pharmacist interventions but simple actions that could be delivered by a lay caregiver. As an example, one study in which the role of the pharmacist was to provide a special medication container to the patient with no further action the score obtained with DEPICT 1 and DEPICT 2 was the same [21]. Another study in which the pharmacist intervention was simply to deliver a medication compliance device to the patient scored similarly in the two versions [22]. These two situations demonstrate that the simpler the intervention, the smaller the difference between the two versions of DEPICT. In cases like these, several domains of the instrument remained in blank, and therefore, the total number of points scored was low. Another explanation for the presence of blank items in some domains of DEPICT can be related to the poor intervention description.

As discussed for the creation of DEPICT 1, the development and validation of a universal tool to characterize clinical pharmacist interventions is a critical step to identify the most powerful components of a complex intervention, that is, components that represent a greater contribution to the outcomes obtained [12]. An improved and more specific version of DEPICT will allow a better discrimination of the intervention components of clinical pharmacy services. Additionally, the retrospective application of DEPICT 2 to pharmacy studies will likely result in a better understanding of the pharmacy service as a whole, facilitating interstudy comparisons and contributing to the reproducibility of the interventions from pharmacy practice studies to the real world. Using DEPICT 2 as a reference guide to reporting pharmacist interventions in future studies could not only be a way of ensuring their reproducibility, but also a way of reducing the heterogeneity obtained in meta-analyses when gathering data from pharmacy studies [4-6]. DEPICT 2 could be considered a new parameter to evaluate biases and increase the applicability of the evidence generated in meta-analyses.

A limitation of our study is that we based the analysis and identification of pharmacist intervention components on the description of services provided by the authors, which could be an issue in studies with poor intervention description. We are aware that efforts are being made to assess the quality of reporting of specific interventions such as those involving a behavioral change [23]. Tools designed for this end are likely more detailed for this type of interventions than DEPICT; however, our aim was to create a valid tool for any type of intervention and not just those behavioral related. Furthermore, the use of these specific tools would be useless to analyse complex interventions comprising both behavioral changes and other types of interventions. As strengths of this study, it can also be said that the subset of RCTs used was international and was based on a comprehensive literature review, and that a high inter-rater reliability was obtained.

In conclusion, DEPICT 2 is an improved version of DEPICT 1 , and it comprises 146 items grouped in 11 domains, as opposed to 
the prior 54-item, 12-domain version. DEPICT 2 allows the analysis of studies describing pharmacists' interventions targeting both patients/caregivers and health care professionals, whereas this distinction was not clearly identifiable in DEPICT 1. DEPICT 2 presents, on average, almost perfect agreement results in the reliability analysis and was proved to better discriminate more components of pharmacist interventions performed as part of clinical pharmacy services. The better discriminating ability of DEPICT 2 will likely ensure consistent reporting of interventions when used in early stages of report preparation and therefore contribute to facilitate the reproducibility of the intervention in clinical practice.

\section{Acknowledgements}

The authors would like to thank Coordenação de Aperfeiçoamento de Pessoal de Nível Superior - CAPES, Ministry of Education, Brazil, for supporting this work (Doctoral Grant reference number BEX 14066/13-0) and the Postgraduate Program in Pharmaceutical Sciences of the Federal University of Parana, Brazil.

\section{References}

1. Chisholm-Burns, M. A., Kim Lee, J., Spivey, C. A., et al. (2010) US pharmacists' effect as team members on patient care: systematic review and meta-analyses. Medical Care, 48 (10), 923-933.

2. Salgado, T. M., Moles, R., Benrimoj, S. I. \& Fernandez-Llimos, F. (2012) Pharmacists' interventions in the management of patients with chronic kidney disease: a systematic review. Nephrology, Dialysis, Transplantation, 27 (1), 276-292.

3. Higgins, J. P. T. \& Green, S. (2011) Cochrane handbook for systematic reviews of interventions version 5.1.0 [updated March 2011]. In The Cochrane Collaboration. Available at: www.cochrane-handbook.org (last accessed 8 October 2014).

4. Charrois, T. L., Zolezzi, M., Koshman, S. L., Pearson, G., Makowsky, M., Durec, T. \& Tsuyuki, R. T. (2012) A systematic review of the evidence for pharmacist care of patients with dyslipidemia. Pharmacotherapy, 32 (3), 222-233.

5. Santschi, V., Chiolero, A., Colosimo, A. L., Platt, R. W., Taffe, P., Burnier, M., Burnand, B. \& Paradis, G. (2014) Improving blood pressure control through pharmacist interventions: a meta-analysis of randomized controlled trials. Journal of the American Heart Association, 3 (2), e000718.

6. Santschi, V., Chiolero, A., Paradis, G., Colosimo, A. L. \& Burnand, B. (2012) Pharmacist interventions to improve cardiovascular disease risk factors in diabetes: a systematic review and meta-analysis of randomized controlled trials. Diabetes Care, 35 (12), 2706-2717.

7. Melchiors, A. C., Correr, C. J., Venson, R. \& Pontarolo, R. (2012) An analysis of quality of systematic reviews on pharmacist health interventions. International Journal of Clinical Pharmacy, 34 (1), 3242.

8. Craig, P., Dieppe, P., Macintyre, S., Michie, S., Nazareth, I. \& Petticrew, M. (2008) Developing and evaluating complex interventions: the new Medical Research Council guidance. British Medical Journal, 337, a1655.

9. Bakker, F. C., Persoon, A., Schoon, Y. \& Olde Rikkert, M. G. (2014) Uniform presentation of process evaluation results facilitates the evaluation of complex interventions: development of a graph. Journal of Evaluation in Clinical Practice, 2014 Oct 14. doi: 10.1111/ jep.12252. [Epub ahead of print]
10. Kennie, N. R., Schuster, B. G. \& Einarson, T. R. (1998) Critical analysis of the pharmaceutical care research literature. Annals of Pharmacotherapy, 32 (1), 17-26.

11. Charrois, T. L., Durec, T. \& Tsuyuki, R. T. (2009) Systematic reviews of pharmacy practice research: methodologic issues in searching, evaluating, interpreting, and disseminating results. Annals of Pharmacotherapy, 43 (1), 118-122.

12. Correr, C. J., Melchiors, A. C., de Souza, T. T., Rotta, I., Salgado, T. M. \& Fernandez-Llimos, F. (2013) A tool to characterize the components of pharmacist interventions in clinical pharmacy services: the DEPICT project. Annals of Pharmacotherapy, 47 (7-8), 946-952.

13. Salgado, T. M., Correr, C. J., Moles, R., Benrimoj, S. I. \& Fernandez-Llimos, F. (2013) Assessing the implementability of clinical pharmacist interventions in patients with chronic kidney disease: an analysis of systematic reviews. Annals of Pharmacotherapy, 47 (11), 1498-1506.

14. Sim, J. \& Wright, C. C. (2005) The kappa statistic in reliability studies: use, interpretation, and sample size requirements. Physical Therapy, 85 (3), 257-268.

15. Landis, J. R. \& Koch, G. G. (1977) The measurement of observer agreement for categorical data. Biometrics, 33 (1), 159-174.

16. Brook, O. H., van Hout, H., Stalman, W., Nieuwenhuyse, H., Bakker, B., Heerdink, E. \& de Haan, M. (2005) A pharmacy-based coaching program to improve adherence to antidepressant treatment among primary care patients. Psychiatric Services, 56 (4), 487-489.

17. Ellis, S. L., Billups, S. J., Malone, D. C., et al. (2000) Types of interventions made by clinical pharmacists in the IMPROVE study. Impact of Managed Pharmaceutical Care on Resource Utilization and Outcomes in Veterans Affairs Medical Centers. Pharmacotherapy, 20 (4), 429-435.

18. Ellis, S. L., Carter, B. L., Malone, D. C., et al. (2000) Clinical and economic impact of ambulatory care clinical pharmacists in management of dyslipidemia in older adults: the IMPROVE study. Impact of Managed Pharmaceutical Care on Resource Utilization and Outcomes in Veterans Affairs Medical Centers. Pharmacotherapy, 20 (12), $1508-1516$

19. Gourley, D. R., Gourley, G. A., Solomon, D. K., et al. (1998) Part 1. Development, implementation, and evaluation of a multicenter pharmaceutical care outcomes study. Journal of the American Pharmaceutical Association (Washington, D.C.: 1996), 38 (5), 567-573.

20. Solomon, D. K., Portner, T. S., Bass, G. E., et al. (1998) Clinical and economic outcomes in the hypertension and COPD arms of a multicenter outcomes study. Journal of the American Pharmaceutical Association (Washington, DC : 1996), 38 (5), 574-585.

21. Rehder, T. L., McCoy, L. K., Blackwell, B., Whitehead, W. \& Robinson, A. (1980) Improving medication compliance by counseling and special prescription container. American Journal of Hospital Pharmacy, 37 (3), 379-385.

22. Schneider, P., Murphy, J. \& Pedersen, C. (2008) Impact of medication packaging on adherence and treatment outcomes in older ambulatory patients. Journal of the American Pharmacists Association: JAPhA, 48 (1), 58-63.

23. Albrecht, L., Archibald, M., Arseneau, D. \& Scott, S. D. (2013) Development of a checklist to assess the quality of reporting of knowledge translation interventions using the Workgroup for Intervention Development and Evaluation Research (WIDER) recommendations. Implement Sci. 2013 May 16; 8, 52.

\section{Supporting Information}

Additional supporting information may be found in the online version of this article at the publisher's web site. 


\section{Appendix A}

\section{Descriptive Elements of Pharmacist Intervention Characterization Tool - DEPICT 2}

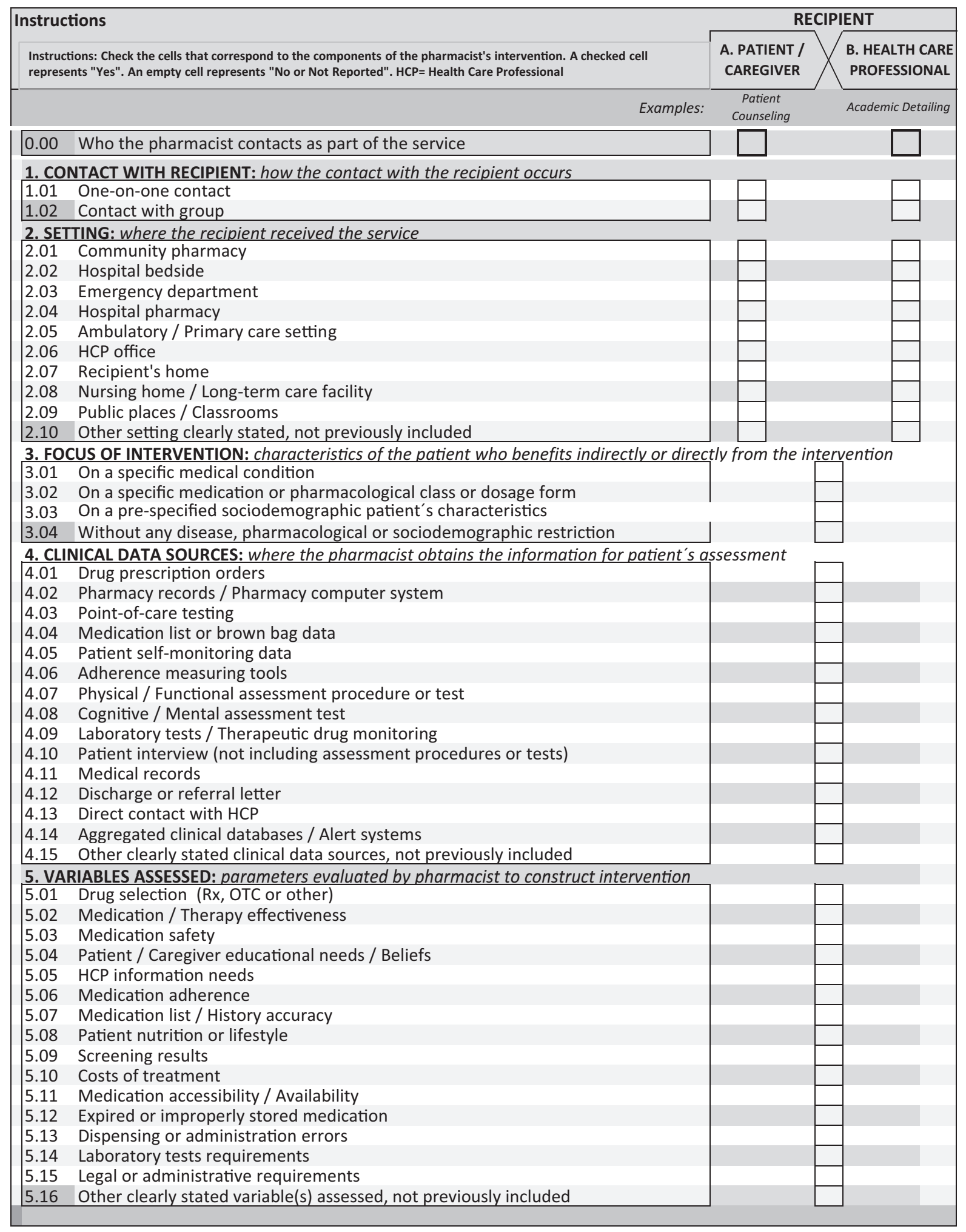




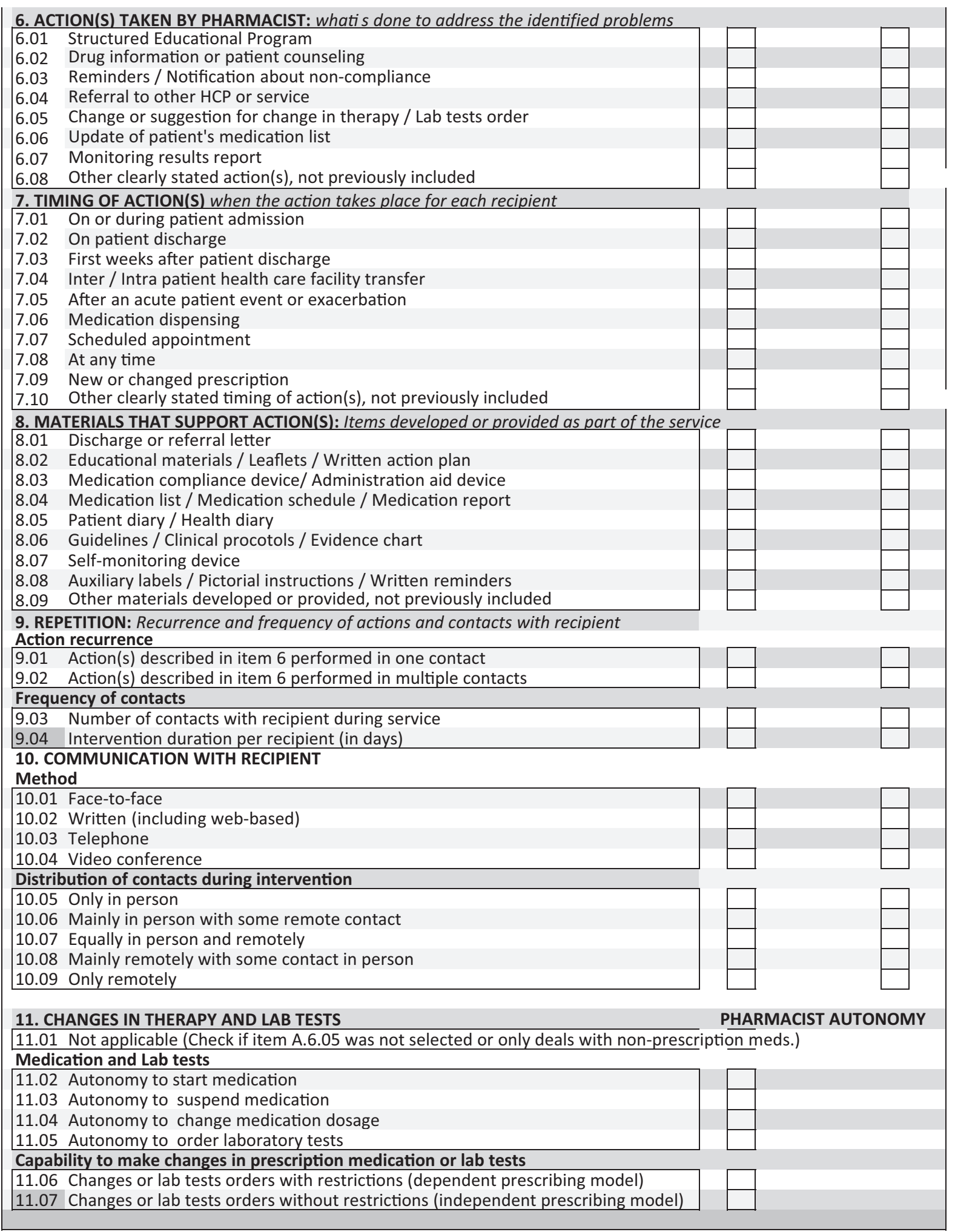

